It is worthwhile noting that the logarithm of the outer factor $p^{r} g(p)$ exists, and is uniquely determined by the magnitudes of the $f_{n}$ on the boundary, since $\log \operatorname{pr}^{r} g(p)$ are uniquely determined by $\log \left|f_{n}(\omega)\right|$.

\title{
REFERENCES
}

1. L. Schwartz, Transformations de Laplace des distributions, Sem. Math. Univ. Lund, Tome Supp. (1952), 196-206.

2. —_ Theorie des distributions, Tome I, II, Hermann, Paris, 1957, 1959.

3. E. J. Beltrami and M. R. Wohlers, Distributional boundary value theorems and Hilbert transforms, Arch. Rational Mech. Anal. 18 (1965), 304-309.

4. K. Hoff man, Banach spaces of analytic functions, Prentice-Hall, Englewood Cliffs, N. J., 1962.

5. A. Friedman, Generalized functions and partial differential equations, PrenticeHall, Englewood Cliffs, N. J., 1963.

6. E. C. Titchmarsh, The theory of functions, 2nd ed., Oxford Univ. Press, Oxford, 1939.

Grumman Aircraft Engineering Company

\section{THREE THEOREMS ON MANIFOLDS WITH BOUNDED MEAN CURVATURE}

\author{
BY F. J. ALMGREN, JR.
}

Communicated by J. Moser, May 27, 1965

The following are three theorems about manifolds having bounded mean curvature which illustrate some of the applications to classical differential geometry of the structure theorems for regular integral varifolds. The proofs, which will appear in $[\mathbf{A}]$, are geometric and measure theoretic. Let $2 \leqq k \leqq n$ be integers.

THEOREM 1. There exist numbers $a(k)>0$ and $b(k, n)<\infty$ with the following property: Let $A$ be a compact k-dimensional manifold of class $C^{2}$ with boundary $B$ and $f: A \rightarrow R^{n}$ be a $C^{2}$ immersion of $A$ into $R^{n}$ having mean curvature no larger than $M$ at each point. If

$$
M^{k}[k \text {-area of } f \mid A] \leqq a(k),
$$

then

$$
[k \text {-area of } f \mid A] \leqq b(k, n)[(k-1) \text {-area of } f \mid B]^{k /(k-1)} .
$$

In particular, if $f$ satisfies the minimal surface equation, then, without additional hypotheses, 


$$
[k \text {-area of } f \mid A] \leqq b(k, n)[(k-1) \text {-area of } f \mid B]^{k /(k-1)} .
$$

THEOREM 2. There exists a number $L<\infty$ with the following property: Let $A$ be a compact $k$-dimensional manifold of class $C^{2}$ having boundary $B$ and $f: A \rightarrow R^{n}$ be a $C^{2}$ immersion of $A$ into $R^{n}$ satisfying the minimal surface equation. Let $p, q \in R^{n}$ with $|p-q| \geqq L$. If

$$
f(B) \subset R^{n} \cap\{x:|x-p| \leqq 1 \text { or }|x-q| \leqq 1\},
$$

then

$$
f(A) \subset R^{n} \cap\{x:|x-p| \leqq 1 \text { or }|x-q| \leqq 1\} .
$$

THEOREM 3. For each $M<\infty$ and $\epsilon>0$ there exists $\delta>0$ with the following property: Let $A$ be a compact k-dimensional manifold of class $C^{2}$ with boundary $B$ and $f: A \rightarrow R^{n}$ be a $C^{2}$ immersion of $A$ into $R^{n}$ having mean curvature no larger than $M$ at each point such that

(1) $[k$-area of $f \mid A] \leqq M$;

(2) $[(k-1)$-area of $f \mid B] \leqq M$;

(3) $f(A) \subset R^{n} \cap\left\{x: \operatorname{dist}\left(x,\left\{y: y^{k+1}=y^{k+2}=\cdots=y^{n}=0\right\}\right) \leqq \delta\right\}$; and

(4) $f(B) \subset R^{n} \cap\left\{x: \operatorname{dist}\left(x,\left\{y: y^{k+1}=y^{k+2}=\cdots=y^{n}=0 \quad\right.\right.\right.$ and $\left.\left.\left.\left(y^{1}\right)^{2}+\left(y^{2}\right)^{2}+\cdots+\left(y^{k}\right)^{2}=1\right\}\right) \leqq \delta\right\}$.

Then, for some integer $Z$,

$$
\begin{gathered}
\mid[k \text {-area of } f \mid A]-Z[k \text {-area of the unit } k \text {-ball }] \mid<\epsilon . \\
\text { REFERENCE }
\end{gathered}
$$

[A] F. J. Almgren, Jr., The theory of varifolds-a variational calculus in the large for the $k$-dimensional area integrand, (to appear).

INSTITUTE For AdVANCEd STUdy 